\title{
Rehabilitation training in artificially heated environment
}

\author{
Ping-Chung Leung* \\ Department of Orthopaedics, Institute of Chinese Medicine, The Chinese University of Hong Kong, Hong Kong
}

Sauna has become a popular club house facility where the dweller enjoys relaxation. Some exercise groups like yoga and Qigong practitioners, are making use of the heated environment to achieve quicker and better results of trainings. Sauna therapy is producing a thermal stress through hyperthermia. The cardiovascular system readily responds by increasing the heart rate which can become double the resting stage within minutes and cardiac output may have a $70 \%$ increase. The body's surface response to heat leads to a $40 \%$ of decrease in peripheral vascular resistance, thus allowing rapid peripheral blood flow which is responsible for greater heat dispersal directly from the skin. The chained physiological reactions of increased cardiac and pulmonary outputs, while blood pressure drops suggest that Sauna could be good for chronic diseases. When active stretching are executed simul- taneously with controlled breathing in a smooth synchronized chain of activities under the individual's free will, a harmonized state of mind reaching the level of meditation follows. Sauna room environment initiates a physiological stat equivalent to moderate exercises. Oigong practice is typical anaerobic training. Both Sauna and Qigong lead to a tranquility of the mind. The unique nature of practicing Qigong in a heated environment is therefore clear. A small pilot study on Qigong practice within the Sauna room showed a higher increase in heart rate which amounted to $30 \%-40 \%$ above the pre-exercise level. The blood pressure checked after Qigong, on the contrary, remained stable or even slightly decreased.

Keywords: Qigong, Sauna, Physical training

\section{INTRODUCTION}

Artificially heated environment refers to Club House facilities like Sauna room or Steam room, and recently, may be any ordinary training venue with artificially elevated temperature. Sauna was probably originated from Finland where a wooden room was heated by an electric stove to a temperature of $70^{\circ} \mathrm{C}-100^{\circ} \mathrm{C}$ and humidity reached 10\%-20\% (Kauppinen, 1989; Valtakari, 1988). Dwellers in a Sauna enjoy the heated environment, relaxed and usually do nothing special. When popularity increases, sophisticated heated environments are created with special heating sources like infrared generators of different intensities, and other rooms are fitted with additional steam currents. Sauna, with its heated environment has become special training or treatment venues.

Today, one may join Sauna physiotherapy classes, Sauna yoga and Sauna Qigong. The colourful varieties are trying to enhance the established training effects of the exercises with a heated environment. The additive effects of Sauna and the anaerobic exercises, through a careful reexamination of the reported individual physiological effects given by Sauna and Qigong (which is chosen as an anaerobic exercise) was explored and the outcome of making use of Sauna and Qigong together would be discussed.

No proper report on the practice of Qigong in a Sauna venue is available. Henceforth, a small pilot observational study was organized among five constant Sauna users, to illustrate the safety and basic physiological changes resulting from the combined practice.

\section{Sauna therapy - physiological responses}

Sauna therapy is producing a thermal stress through hyperthermia. The cardiovascular system readily responds by increasing the heart rate which can become double the resting stage within minutes and cardiac output may have a $70 \%$ increase. The body's surface response to heat leads to a $40 \%$ of decrease in peripheral vascular resistance, thus allowing rapid peripheral blood flow which is responsible for greater heat dispersal directly and through sweating from the skin. At the same time blood pressure, both systolic
${ }^{*}$ Corresponding author: Ping-Chung Leung (iD https://orcid.org/0000-0002-0195-4688 Department of Orthopaedics, Institute of Chinese Medicine, The Chinese University of Hong Kong, 5/F School of Public health Building, Prince of Wales Hospital, Shatin, Hong Kong

Tel: +85-222528868, Fax: +85-226325441, E-mail: pingcleung@cuhk.edu.hk

Received: March 31, 2017 / Accepted: October 8, 2017
This is an Open Access article distributed under the terms of the Creative Commons Attribution Non-Commercial License (http://creativecommons.org/licenses/by-nc/4.0/) which permits unrestricted non-commercial use, distribution, and reproduction in any medium, provided the original work is properly cited. 
and diastolic drop, consequently blood circulation to muscles and internal organs also drop. An acute increase in metabolic rate and oxygen consumption follows: overall effects are similar to moderate exercises (Kauppinen, 1989; Kukkonen-Harjula et al., 1989).

The circulatory effects also affects respiratory physiology since pulmonary blood flow is increased, facilitating pulmonary function like forced vital capacity and expiratory flow rate (Kiss et al., 1994).

The sympathetic hypothalamic - pituitary - adrenal axis respond by adjusting the relevant hormonal production resulting in stabilizing fluid balance; muscle and tendon relaxations (via proprioceptive harmonization); and pain control with relaxation of the mind (via endorphins) (Crinnion, 2011; Hasan et al., 1967; Kauppinen et al., 1989).

The chained physiological reactions could be so positive that advocates on Sauna bath have recommended it for the actual treatment of various chronic diseases like essential hypertension; early congestive heart failure, depression, arthritis, and chronic fatigue syndrome (Crinnion, 2011).

\section{Qigong practice - physiological responses}

Qigong training involves three major components, namely stretching of limbs, controlled respirations and meditation.

Limb stretching involves the pulling of muscles, ligaments, tendons and joints, the attachments of which are provided with rich proprioceptive sensory receptors. Stretching of these structures therefore is providing powerful proprioceptive neurosensory input which initiates chain reactions of pain control and harmonizing effects (Gate theory) (Leung, 2011; Melzack and Wall, 1996). Even when the small joints of fingers are forcefully stretched, the same physiological effects are expected.

Controlled respiratory breathings are practiced simultaneously with the stretching exercises. The pattern of respiration is intentionally converted to longer inspirations and extralong expirations, making full use of abdominal and diaphragmatic muscles, coupled with tightening of the anal sphincter (Leung, 2011).

When active stretching are executed simultaneously with controlled breathing in a smooth synchronized chain of activities under the individual's free will, a harmonized state of mind reaching the level of meditation follows.

During the Qigong training, the intention is to give a good rest to the central nervous system: free it from motor and sensory burdens, relieve it from complex memories, emotions and problem solving needs (Li and Chu, 2008).

Research on the immediate physiological responses to Qigong practice are limited. Generally speaking, the active stretching and controlled breathing would initiate active circulatory responses manifested as a steady limited increase in heart rate and blood pressure. The increase is observed to downgrade on more frequent trainings.

Research on the long termed effects of Qigong practice are plentiful, and have given vivid demonstration of improved heartlung fitness, musculoskeletal strength and quality of life improvements (Chang et al., 2006; Du et al., 2006).

Psychologists are very much interested to study the cognitive and mental state of Qigong practitioners. Both the supportive and therapeutic results are very positive. Properly conducted clinical trials on volunteers practicing meditative breathing, using neuro-electrophysiological tools confirmed the fostering of a relaxed and attentive mind in the electroencephalography pattern changes (Chan et al., 2011a). The same group of researchers conducted a randomized controlled trial on volunteers with Depressive Mood treated with meditative breathing. A short term serious training of only four weeks resulted in improvement (Chan et al., 2011b).

\section{What could be expected when Sauna and Qigong are combined?}

The physiological implications of both Sauna and Qigong have been so clearly beneficial that one wonders whether practicing Qigong in a Sauna room would achieve extra merits. Sauna room has only limited space which does not allow proper exercises. Skepticism against Sauna could just firmly insist on this bias. Qigong practice, when carefully adopted, could be fulfilled in a venue with extremely limited space. However, Qigong is recommended for lengthy practice in order to achieve the maximal benefits. If Qigong is practiced within a Sauna room, 10- to 15-min stay would be the standard because longer stay becomes intolerable. Within this short period, because of the heated environment, the flourishing circulatory state would be reached beyond any level that Qigong practice would be able to initiate. The controlled respiration component of Qigong at the same time would be enhanced by the facilitated pulmonary circulation in the hyperthermia environment. If both hyperthermia and Qigong meditation are leading towards a tranquility of mind, it is logical to assume that this mental state of harmony could be better guaranteed when the practitioner uses them together and combined.

Qigong practice is typically anaerobic, not affecting much the metabolic rate of the whole body. In contrast, hyperthermia initiates the chained reactions of hormonal adjustments along the hypothalamic - pituitary - adrenal axis which lead to an acute eleva- 
Table 1. Pilot study showing changes in blood pressure and heart rate before and after Sauna Oigong

\begin{tabular}{|c|c|c|c|c|c|c|c|c|}
\hline \multirow{2}{*}{ Subject } & \multicolumn{2}{|c|}{ Before Sauna } & \multicolumn{2}{|c|}{ After Sauna } & \multicolumn{2}{|c|}{ Before Sauna + Qigong } & \multicolumn{2}{|c|}{ After Sauna + Qigong } \\
\hline & $\mathrm{HR}^{\mathrm{a})}$ & $\mathrm{BP}^{\mathrm{a})}$ & $H R^{\text {a) }}$ & $\mathrm{BP}^{\mathrm{al}}$ & $H R^{b)}$ & $\mathrm{BP}^{\mathrm{bl}}$ & $H \mathrm{R}^{\mathrm{bl}}$ & $\mathrm{BP}^{\mathrm{bl}}$ \\
\hline 1 & 55 & 130/88 & 62 & $125 / 80$ & 53 & $128 / 90$ & 82 & $120 / 78$ \\
\hline 2 & 59 & $128 / 80$ & 68 & $125 / 78$ & 57 & $129 / 85$ & 88 & $118 / 72$ \\
\hline 3 & 65 & $135 / 90$ & 69 & $138 / 92$ & 64 & $135 / 90$ & 90 & $136 / 95$ \\
\hline 4 & 58 & $127 / 80$ & 63 & $120 / 70$ & 60 & $127 / 80$ & 85 & $126 / 85$ \\
\hline 5 & 68 & $140 / 85$ & 75 & $140 / 80$ & 69 & $140 / 85$ & 95 & $138 / 90$ \\
\hline
\end{tabular}

${ }^{a}$ Heart rate (HR) and blood pressure (BP) changes after Sauna (data were averaged from 3-day records). ${ }^{b} H R$ and BP changes after Sauna and Qigong practice (data were averaged from 3-day records).

tion in oxygen consumption and increased metabolic rate, described to be equivalent to moderate physical exercises (Kauppinen, 1989; Kukkonen-Harjula et al., 1989). This might mean the Qigong practice in a hyperthermic environment might be achieving both anaerobic and aerobic benefits.

\section{MATERIALS AND METHODS}

A pilot study on the immediate effects of Qigong practice in a Sauna room is designed. The aim was to study the safety and short-termed cardiovascular effects of practicing Qigong inside a Sauna room. Five volunteers who had more than 2-year experience using the Sauna as a recreational activity was recruited. They were given instructions on a simple package of Qigong procedures consisting of a variety of upper limbs stretching, bent knees static standing and controlled breathing using abdominal and diaphragmatic muscles simultaneously with tightening of anal sphincter.

After careful evaluation of their standard performance, usually on completion of self-practice for a week, the volunteers were asked to go for their usual Sauna recreation without exercises for $15 \mathrm{~min}$. On separate days they practiced Qigong while staying in the Sauna room for $15 \mathrm{~min}$. Radial pulse and blood pressure were checked before and after each mode of Sauna practice. The procedures were repeated 3 times for each volunteer on different dates and the average measurements were taken for assessment.

\section{RESULTS}

The five experiences Sauna users did not feel any discomfort or unusual feeling after doing Qi-gong practice inside the Sauna room.

The cardiovascular responses within the Sauna room without Qigong were very similar to published reports. Heart rate increase was felt among all volunteers soon after entry. On completion of 15 min of stay an increase of about $15 \%-17 \%$ was common to all five volunteers. The blood pressure never rose but instead dropped slightly or remained stable after $15 \mathrm{~min}$.

Practicing stretching and controlled breathing in the Sauna room produced a higher increase in heart rate which amounted to $30 \%-40 \%$ above the pre-exercise level. The blood pressure checked after the Qigong exercise, on the contrary, remained stable or even slightly decreased which listed in Table 1.

Volunteers were asked whether stretching and controlled breathing inside the heated Sauna imposed any symptoms. All of them did not experience respiratory or musculoskeletal discomfort.

\section{DISCUSSION}

At the entrance to a Sauna room one often notices a warning plague: "People with hypertensive and heart diseases are not advised to enter". The safety of the Sauna bath therefore, has been a general concern.

Is hyperthermia exposure really dangerous? If so, doing exercises in the Sauna room should really been condemned.

Such death as a result of heart attack did occur in the Sauna room. But reliable epidemiological studies have shown that sudden cardiac death actually happened more often in any other environment (Kenttämies and Karkola, 2008). Actually in the Scandinavian countries where Sauna is most popular, there are suggestions that cardiac deaths are less common (Crinnion, 2011). Nevertheless, cardiovascular patients suffering from acute symptoms, of course need to be vigilant.

Now that the unique influences of hyperthermia, pushing the cardiovascular system to respond passively in a direction beneficial to heart-lung function and nurturing the neurological system to a somatic-autonomic harmony, there need to be more active explorations on the practical clinical use of the Sauna environment. On the other hand, the oriental practice of Qigong (also yoga) has also gained sufficient trusts on the same lines (National Centre for Complementary and Integrative Health, 2014), combining the 
hyperthermic recreation with meditative controlled breathing exercises could be a simple way to achieve synergistic effects. The unique nature of the quick response and stringency of space requirement would be particularly favourable for the busy individuals who have tight working schedules and those who are unsuitable for aerobic exercises in wide fields.

\section{CONFLICT OF INTEREST}

No potential conflict of interest relevant to this article was reported.

\section{ACKNOWLEDGMENTS}

Authors are indebted to the Ming Lai Foundation support given to the team responsible for the study.

\section{REFERENCES}

Chan AS, Cheung MC, Sze SL, Leung WW, Shi D. Shaolin dan tian breathing fosters relaxed and attentive mind: a randomized controlled neuro-electrophysiological study. Evid Based Complement Alternat Med 2011a;2011:180704.

Chan AS, Cheung MC, Tsui WJ, Sze SL, Shi D. Dejian mind-body intervention on depressive mood of community-dwelling adults: a randomized controlled trial. Evid Based Complement Alternat Med 2011b;2011:473961.

Chang WC, Zhung TB, Wu CH. Qi-gong and cognitive changes. Chin J Behav Med Sci 2006;15:827-828.

Crinnion WJ. Sauna as a valuable clinical tool for cardiovascular, autoimmune, toxicant- induced and other chronic health problems. Altern Med Rev 2011;16:215-225.

Du SW, Chang CL, Wang S. Qi-gong and cardiac function of middle and old age people. Chin J Sports Med 2006;25:721-726.

Hasan J, Karvonen MJ, Piironen P. Special review. II. Physiological effects of extreme heat. As studied in the Finnish "sauna" bath. Am J Phys Med 1967;46:1226-1246.

Kauppinen K. Sauna, shower, and ice water immersion. Physiological responses to brief exposures to heat, cool, and cold. Part II. Circulation. Arctic Med Res 1989;48:64-74.

Kauppinen K, Pajari-Backas M, Volin P, Vakkuri O. Some endocrine responses to sauna, shower and ice water immersion. Arctic Med Res 1989;48:131-139.

Kenttämies A, Karkola K. Death in sauna. J Forensic Sci 2008;53:724-729.

Kiss D, Popp W, Wagner C, Zwick H, Sertl K. Effects of the sauna on diffusing capacity, pulmonary function and cardiac output in healthy subjects. Respiration 1994;61:86-88.

Kukkonen-Harjula K, Oja P, Laustiola K, Vuori I, Jolkkonen J, Siitonen S, Vapaatalo H. Haemodynamic and hormonal responses to heat exposure in a Finnish sauna bath. Eur J Appl Physiol Occup Physiol 1989;58:543-550.

Leung PC. Natural healing in Chinese Medicine: Qi-gong and Tai Chi. In: Leung PC. Healthy aging. Singapore: World Scientific Publishing; 2011. p. 221-244.

Li SC, Chu WC. Recent scientific research on Qi-gong in Japan. Rep Relat Chin Med Outside China 2008;25:276-279.

Melzack R, Wall PD. Peripheral nerve and spinal mechanisms. In: Melzack R, Wall PD. The challenge of pain, 2nd ed. London: Penguin Books; 1996. p. 81-107.

National Centre for Complementary and Integrative Health (NCCIH). Yoga for Health [Internet]. Bethesda (MD): National Centre for Complementary and Integrative Health; 2014 Oct [cited 2017 Feb 1]. Available from: https://nccih.nih.gov/health/providers/digest/yoga.

Valtakari P. The sauna and bathing in different countries. Ann Clin Res 1988;20:230-235 